\title{
Variational Region-Based Segmentation Using Multiple Texture Statistics
}

\author{
Imen Karoui, Ronan Fablet, Jean-Marc Boucher, Senior Member, IEEE, and Jean-Marie Augustin
}

\begin{abstract}
This paper investigates variational region-level criterion for supervised and unsupervised texture-based image segmentation. The focus is given to the demonstration of the effectiveness and robustness of this region-based formulation compared to most common variational approaches. The main contributions of this global criterion are twofold. First, the proposed methods circumvent a major problem related to classical texture based segmentation approaches. Existing methods, even if they use different and various texture features, are mainly stated as the optimization of a criterion evaluating punctual pixel likelihoods or similarity measure computed within a local neighborhood. These approaches require sufficient dissimilarity between the considered texture features. An additional limitation is the choice of the neighborhood size and shape. These two parameters and especially the neighborhood size significantly influence the classification performances: the neighborhood must be large enough to capture texture structures and small enough to guarantee segmentation accuracy. These parameters are often set experimentally. These limitations are mitigated with the proposed variational methods stated at the region-level. It resorts to an energy criterion defined on image where regions are characterized by nonparametric distributions of their responses to a set of filters. In the supervised case, the segmentation algorithm consists in the minimization of a similarity measure between region-level statistics and texture prototypes and a boundary based functional that imposes smoothness and regularity on region boundaries. In the unsupervised case, the data-driven term involves the maximization of the dissimilarity between regions. The proposed similarity measure is generic and permits optimally fusing various types of texture features. It is defined as a weighted sum of Kullback-Leibler divergences between feature distributions. The optimization of the proposed variational criteria is carried out using a level-set formulation. The effectiveness and the robustness of this formulation at region-level, compared to classical active contour methods, are evaluated for various Brodatz and natural images.
\end{abstract}

Index Terms-Active regions, level sets, nonparametric distributions, supervised and unsupervised segmentation, texture similarity measure.

Manuscript received January 04, 2008; revised March 26, 2010; accepted March 26, 2010. Date of publication September 02, 2010; date of current version November 17, 2010. The associate editor coordinating the review of this manuscript and approving it for publication was Dr. Ying Wu.

I. Karoui, R. Fablet and J.-M. Boucher are with the Telecom Bretagne, UMR CNRS Lab, 29238 Brest Cedex 3, France (e-mail: imen.karoui@telecom-bretagne.eu; ronan.fablet@telecom-bretagne.eu; jm. boucher@telecom-bretagne.eu).

I. Karoui and J.-M. Augustin are with the Institut Français de Recherche et dExploitation de la Mer, 29280 Plouzane, France (e-mail: jean-marie. augustin@ifremer.fr).

Color versions of one or more of the figures in this paper are available online at http://ieeexplore.ieee.org.

Digital Object Identifier 10.1109/TIP.2010.2071290

\section{INTRODUCTION}

$\mathbf{T}$ EXTURE describes a visual information which is related to local spatial variations of color, orientation and intensity in an image. It is usually described by qualitative adjectives such as smooth or rough, coarse or fine, homogeneous or random, etc. This information is fundamental in image analysis and interpretation and the segmentation of an image into homogeneous regions, in terms of textural features, remains a complex issue. An effective and efficient texture segmentation method is of key interest in numerous domains such as biomedical image analysis, industrial inspection, analysis of remote sensing images, sonar or aerial imagery [1]-[4], etc.

Pixel-based and region-based techniques can be seen as the two major categories of approaches for image segmentation. Whereas pixel-based schemes, such as k-means [5], standard Markov random fields [6], [7], consider image segmentation as a labeling issue at pixel-level, region-based approaches directly search for a relevant image partition. The main methods belonging to this second category are split-and-merge technique [8], [9], region-based Bayesian segmentation [10], active contours or deformable models [11]-[13]. As far as texture segmentation is concerned, region-based techniques appear more adapted, since texture characteristics are by nature nonlocal characteristics. Region-based approaches, especially active contours associated with a level-set setting, offer an efficient manner to cope with texture and geometrical features at the region-level. Recently, there has been a considerable amount of work that places texture segmentation in the framework of optimization theory using curve evolution techniques [2], [11], [14]-[17]. The reported results are very encouraging. In general, these methods state the segmentation problem as the minimization of an energy $E=E_{1}+E_{2}$ composed of a data-driven term, denoted by $E_{1}$, and a regularization term denoted by $E_{2}$. The goal is to determine the image partition $\left\{\Omega_{k}\right\}_{k=1: K}$ that minimizes $E$, where $\left\{\Omega_{k}\right\}_{k}$ are image regions. Formally, the segmentation problem is expressed as follows:

$$
\left\{\Omega_{k}\right\}_{k=1: K}=\arg \min E, \Omega_{k} \bigcap_{k \neq k^{\prime}} \Omega_{k^{\prime}}=\phi
$$

Texture segmentation based upon active contour techniques involve two main categories of approaches.

- Methods that rely on the optimization either of information theoretical criteria mainly the entropy and the mutual information or of the product of pixel likelihoods. These methods assume that textural features are independent and identically distributed (i.i.d.) [11], [14]-[17]. 


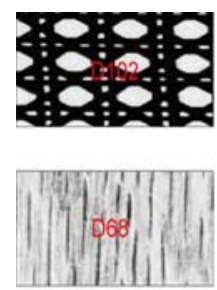

(a)

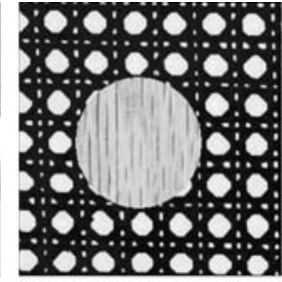

(b)

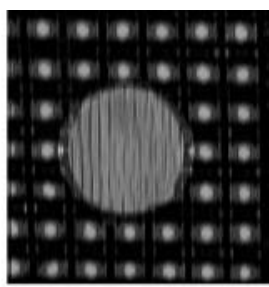

(c)
Fig. 1. Two-class Brodatz mosaic. (a) Considered Brodatz textures D102 and D68. (b) Two-class mosaic. (c) Response of the mosaic to a Gabor filter.

- Methods that exploit image neighborhoods and optimize criteria based upon neighborhood statistics [2], [3], [18], [19].

Without loss of generality, the data-term $E_{1}$ associated to all these methods can be expressed as follows:

$$
E_{1}=\sum_{k=1}^{K} \int_{\Omega_{k}} \Psi\left(p_{k}\right) d s
$$

where $p_{k}$ is the punctual likelihood. For the first category, this likelihood depends only upon pixel feature: $p_{k}(s)$. For the second category, it depends upon feature distribution in the pixel's neighborhood (that we denote by $\left.W_{s}\right): p_{k}\left(s, W_{s}\right)$. $\Psi$ is a function that defines the optimized criterion:

- $\Psi(x)=\log (x)$ for likelihood maximization based criterion;

- $\Psi(x)=x \log (x)$ for entropy based criterion;

- $\Psi(x)=\operatorname{dist}(x)$ for distance based criterion, where dist is a metric.

The existing methods differ in the computation of the probability $p_{k}$. Some methods are based upon parametric models. In [14], pixel likelihoods $\left\{p_{k}(s)\right\}$ are computed according to a Gaussian mixture of filter response statistics (Gaussian and Gabor filters). In [11] and [15], the distribution of texture features (wavelet coefficient) are modeled by generalized Gaussian models. In [19], generalized Laplacian models are considered to describe the statistics of various linear filters (the discrete cosine transform, steerable pyramids, and various orthogonal wavelets). In [17], Gaussian distributions are used to model feature channels extracted from structure tensor, etc. Other approaches rely on nonparametric distributions to evaluate pixel likelihoods such as in [18], where nonparametric neighborhood statistics were combined to an entropy-based metric. In [3], Kadir et al. use a nonparametric model for image intensities and in [20], the authors use nonparametric statistics to optimize image entropy and mutual information, etc.

These methods work well when texture feature distributions $p_{k}$ are disjointed and may fail when these distributions significantly overlap. Fig. 1 shows an example of a mosaic composed of two Brodatz textures [21]: D102 and D68. D102 has a coarse and regular periodic texture and D68 a weakly ordered one (wood grain). These two textures depict overlapping histograms of their response to a Gabor filter (Fig. 2). Approaches based upon the punctual likelihood $p_{k}(s)$ [11], [14]-[17] computed from these histograms cannot correctly segment such an image. Feature distributions depict an overlap corresponding to bright patches. As the local likelihood or similarity of these bright

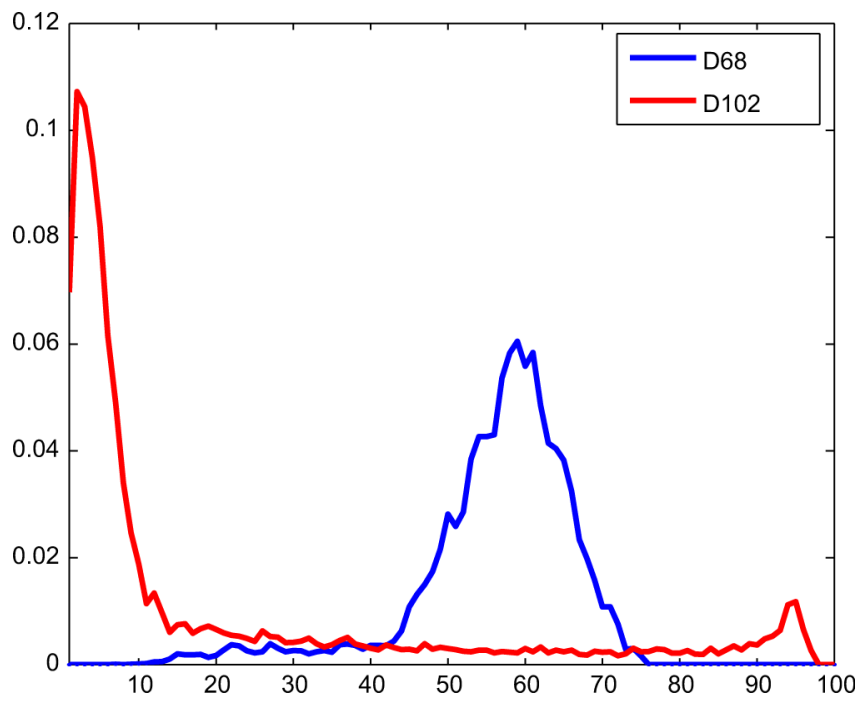

Fig. 2. Histograms of the response of the two textures (D102 and D68) to a Gabor filter.

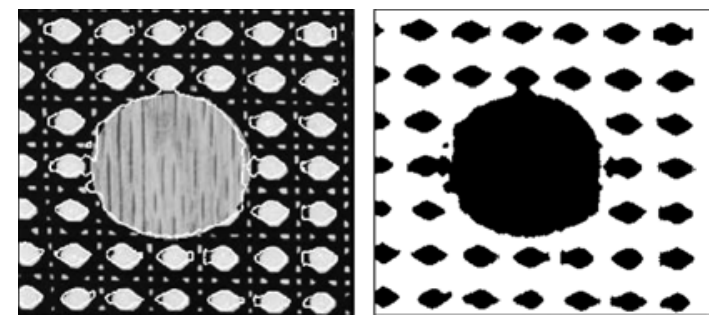

Fig. 3. Segmentation of the two-class mosaic (Fig. 1) by a classical variational approach [11], [14], [15] relying on the computation of pixel-level likelihoods of the Gabor feature.

patches in texture D102 is maximal for the model extracted form texture D68, these patches are misclassified as shown in Fig. 3. This example also stresses the dependence upon the choice of the analysis window when criterion $E_{1}$ actually exploits information in pixel neighborhoods $\left\{W_{s}\right\}$ [19] (Fig. 4). A $3 \times 3$ analysis window (neighborhood) is not large enough to capture the structures of texture D102, whereas a $33 \times 33$ window leads to inaccurate boundaries. $T_{W}=21 \times 21$ seems to be a good tradeoff. In the general case, it is however difficult to determine an optimality criterion for the choice of the analysis window especially when the image is composed of several textures, each with a given coarseness.

The approach proposed in this paper addresses these issues as illustrated in Fig. 5. Our method uses a global criterion that combines the advantages of an efficient texture characterization with global region variational methods. The two key features are: a region-level characterization using nonparametric texture statistics and the definition of a variational observation-driven criterion from a texture-based similarity measure at region-level. Following recent works [4], [9], [22]-[24], we consider texture features computed as nonparametric statistics of filter outputs w.r.t. a varied set of filters. Observation-driven term $E_{1}$ is then defined as

$$
E_{1}=\sum_{k=1}^{K} \sum_{f} w_{f} S\left(Q_{f}^{k}, D_{f}\left(\Omega_{k}\right)\right)
$$




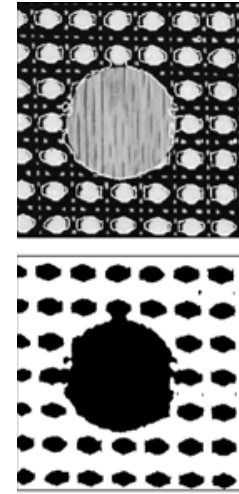

(a)
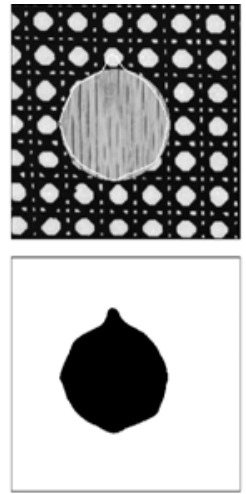

(b)

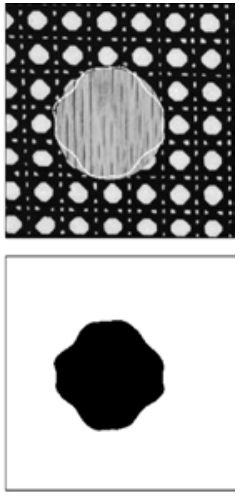

(c)
Fig. 4. Segmentation of the two-class mosaic (Fig. 1) by a classical approach [19] exploiting pixel neighborhood for different sizes of square analysis windows $T_{W}$ (a) $T_{W}=3 \times 3$ (b) $T_{W}=21 \times 21$ (c) $T_{W}=33 \times 33$.

where $S$ is a similarity measure. $Q_{f}^{k}$ refers to the considered texture statistics estimated from training samples for class $k$ and feature $f$ and $D_{f}\left(\Omega_{k}\right)$, the observed global texture statistics for region $\Omega_{k}$ for feature $f$.

Energy $E_{1}$ is minimum when the segmented regions depict texture statistics similar to prototype models. In contrast to the pixel-level formulation, our energy cannot be broken down as a sum of potential over image sites and a given site $s$ contributes to the proposed data-driven term through its participation to the computation of region-level statistics.

The gradient-based minimization of $E_{1}$ exploits the shape derivative tools introduced in [25]. In [25], the Gâteau derivative was applied to a two class image segmentation based upon matching intensity histograms using Hellinger distance. The paper is organized as follows. Texture features and the similarity measure detailed in our previous work [26], [27] are briefly introduced in Section II. The proposed supervised segmentation criterion and its differences with classical ones are detailed in Section III. Its generalization to unsupervised case is described in Section IV. Experiments and comparisons with classical variational approaches are reported and discussed in Section V and conclusions are outlined in Section VI.

\section{TeXture BASED Similarity MEASURE}

Many texture features have been proposed in the literature. Co-occurrence matrices, wavelet frames, quadrature mirror filter-banks and Gabor filters have been shown to be the most effective descriptors [28]. But none of these feature classes outperforms the others for all texture categories. Each feature computed for certain parameters may emphasize particular texture characteristics (scale, direction, smoothness, periodicity, etc.). Here as described in our previous work [26] dealing with supervised texture discrimination, we use a large set of various features computed for different parameters and we select the most discriminating ones. The following set of textural filters is considered:

- 121 co-occurrence distributions with parameters $\left\{\left(d_{x}, d_{y}\right) \in\{1, \ldots, 10\} \times\{1, \ldots, 10\}\right\}$, where $d_{x}$ and
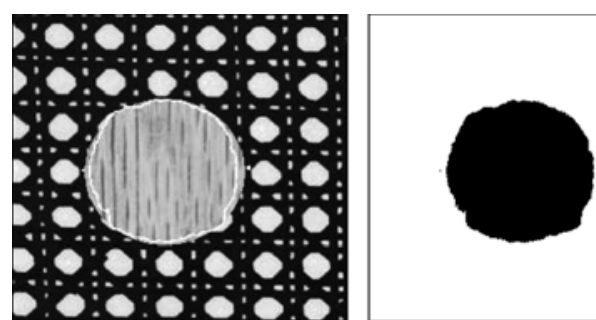

Fig. 5. Segmentation of the two-class mosaic (Fig. 1) by the proposed method.

$d_{y}$ refers, respectively, to the horizontal and vertical displacement;

- 50 distributions of the magnitude of Gabor filter responses, computed for six radial frequencies $\left\{\sqrt{2} / 2^{k}\right\}_{k=1: 6}$, and five orientations: $\left\{0^{\circ}, 25^{\circ}, 45^{\circ}, 90^{\circ}, 135^{\circ}\right\}$;

- 48 distributions of the energy of the image wavelet packet coefficient computed for different bands (we used different wavelet types: Haar, Daubechies and Coiflet).

Formally, we characterize each texture sample $T$ by a set of the $F$ first-order statistics of its responses to the predefined filters $\left\{D_{f}(T)\right\}_{f=1: F}$. Note that $F$ accounts both for given filters and associated parameterizations. Subsequently, index $f$ will refer to a given filter category with some parameterization. Given reference texture statistics $Q^{k}=\left\{Q_{f}^{k}\right\}_{f=1: F}$, the similarity measure $K L_{w}\left(Q^{k}, D(T)\right)$ between texture sample $T$ and the texture class indexed by $k$ is defined as

$$
K L_{w}\left(Q^{k}, D(T)\right)=\sum_{f=1}^{F} w_{f}^{2} K L\left(Q_{f}^{k}, D_{f}(T)\right)
$$

where $K L$ is the Kullback-Leibler divergence [29] and weights $w_{f}$ verify $\sum_{f=1}^{F} w_{f}^{2}=1$. The estimation of the weights $\left\{w_{f}^{2}\right\}$ can be issued from the maximization of a supervised margin criterion detailed in [26].

\section{LeVel Set Segmentation}

We detail in the following the proposed region-level segmentation framework. Regarding the curvature-driven regularization criteria $\left(E_{2}\right)$ and the multiclass image partition constraints, we use classical functionals detailed in [27]. The numerical implementation relies on is based upon a level set setting [30]. The proposed approach relies on the definition of the data-term $E_{1}$ at region-level according to the similarity measure $K L_{w}$ between reference statistics $Q^{k}$ and region statistics $D\left(\Omega_{k}\right)=$ $\left\{D_{f}\left(\Omega_{k}\right)\right\}_{f=1: F}$ :

$$
E_{1}\left(\left\{\Omega_{k}\right\}_{k=1: K}\right)=\sum_{k=1}^{K}\left|\Omega_{k}\right| K L_{w}\left(Q^{k}, D\left(\Omega_{k}\right)\right)
$$

where, $D\left(\Omega_{k}\right)=\left\{D_{f}\left(\Omega_{k}\right)\right\}_{f=1: F}$ and $D_{f}\left(\Omega_{k}\right)$ is the marginal distribution of the image response to the filter indexed by $f$ estimated on the region $\Omega_{k}$.

Using the Gâteaux derivative introduced in [25], the evolution equations of the level set functions denoted by $\left\{\varphi_{k}\right\}_{k}$ [27] 
associated to the energy $E_{1}$ are the following (see Appendix A for details):

$$
\begin{aligned}
\frac{\partial \varphi_{k}}{\partial t}(s, t) & =[\underbrace{-K L_{w}\left(Q^{k}, D\left(\Omega_{k}\right)\right)}_{\text {Global term }} \\
& +\underbrace{\sum_{f=1}^{F} w_{f}^{2}\left(\frac{Q_{f}^{k}}{D_{f}\left(\Omega_{k}\right)} * g_{\sigma}\left(h_{f}(s)\right)-1\right)}_{\text {Local term }}]\left|\nabla \varphi_{k}\right|
\end{aligned}
$$

where $h_{f}$ is the filter response indexed by $f$ (for instance, for a co-occurrence matrix with parameters $\mu=(d, \theta)$, $h_{f}: \Omega \rightarrow[1, N g] \times[1, N g]$ and $h_{f}(s)=(I(s), I(s+\mu))$ where $I(s)$ is the gray value at pixel $s$ and $N g$ the total gray level number), $g_{\sigma_{f}}$ is a centered Gaussian kernel with standard deviation $\sigma_{f}$ and $*$ denotes the convolution symbol.

In contrast to classical functionals related to classical functionals described in Section I (2) which are of the form

$$
\frac{\partial \varphi_{k}}{\partial t}=\left|\nabla \varphi_{k}\right| \Psi\left(p_{k}\right), \quad \forall k \in\{1: K\}
$$

the evolution equations related to the proposed method involve a global term and a local one.

- The global term $-K L_{w}\left(Q^{k}, D\left(\Omega_{k}\right)\right)$ : this term is always negative or null. It is a contraction force that reduces the size of heterogeneous regions: the lower the heterogeneity, the lower the force. This term will avoid to converge towards a local minimum that doses not give regions with the correct statistics mainly on multimodal features (see videos http://public.enst-bretagne. fr/rfablet/Demos/demoIKaroui/classic.avi and http:// public.enst-bretagne.fr/rfablet/Demos/demoIKaroui/proposed.avi)

- The local term

$$
\sum_{f=1}^{F} w_{f}^{2}\left(Q_{f}^{k} / D_{f}\left(\Omega_{k}\right) * g_{\sigma}\left(h_{f}(s)\right)-1\right):
$$

this term compares locally the feature values at each pixel $s$. This term can be positive or negative and tends to locally fit region statistics to the prototype statistics. The contribution of each descriptors $f$ is weighted by $w_{f}^{2}$.

We use as level set functions $\left\{\varphi_{k}\right\}_{k=1: K}$ signed distance functions. The initial segmentation is computed from a moving window approach: each image pixel $s$ is characterized by a set of features estimated on an analysis window centered at $s$ that we denote by $W_{s}$ and the initial pixel-based classification is given by

$$
\operatorname{label}(\mathrm{s})=\arg \min _{k} K L_{w}\left(Q^{k}, D\left(W_{s}\right)\right)
$$

where $D\left(W_{s}\right)=\left\{D_{f}\left(W_{s}\right)\right\}_{f=1: F}$ is the set of distributions associated to the different filters which are estimated locally within the neighborhood of pixel $s$.

\section{UNSUPERVISED TEXTURE SEGMENTATION}

For the unsupervised case, proposed approaches are also usually based upon energy criterion evaluated as a sum of punctual statistics [20], [31]. The most popular criteria are the entropy or mutual information maximization [20], [31] and likelihood maximization alternating class feature estimation and segmentation [13], [17], [32]. Here, we use the region based similarity $K L_{w}$ to define an unsupervised segmentation criterion that consists in the maximization of the similarity measure between regions

$$
E^{1}\left(\left\{\Omega_{k}\right\}_{k=1: K}\right)=\sum_{\left(k, k^{\prime}\right), k^{\prime} \neq k}^{K} K L_{w}\left(D\left(\Omega_{k}\right), D\left(\Omega_{k}^{\prime}\right)\right) .
$$

For the unsupervised case two strategies might be considered: a uniform prior, $w_{f}^{2}=1 / F, \forall f$, or iterations between regionlevel image segmentation and the estimation of weights $\left\{w_{f}^{2}\right\}$ from segmented regions.

We showed (Appendix B) that the proposed criterion allows to separate the image into homogeneous regions. Using the Gâteau derivative tools, the evolution equations associated with the proposed criterion in (10), shown at the bottom of the page. These evolution equations, involve a global term and a local one.

- Global term $-\sum_{k^{\prime}, k^{\prime} \neq k}^{K} K L_{w}\left(D\left(\Omega_{k}\right), D\left(\Omega_{k^{\prime}}\right)\right)$ : the larger the dissimilarity between the region and the others, the larger the contraction force: this term will penalize the expansion of the regions when the dissimilarities increase. This global constraint does not appear in classical approaches [20], [31].

- Local term:

$$
\sum_{k^{\prime}, k^{\prime} \neq k}^{K} \sum_{f=1}^{F} w_{f}^{2}
$$

$\left(\log D_{f}\left(\Omega_{k}\right) / D_{f}\left(\Omega_{k^{\prime}}\right)-D_{f}\left(\Omega_{k^{\prime}}\right) / D_{f}\left(\Omega_{k}\right)+1\right)$

$* g_{\sigma_{f}}\left(h_{f}(s)\right)$. This term is quite similar to those of classical approaches. It compares punctual pixel likelihoods for the different regions: it tends to assign the pixel to the region with the maximal likelihood.

$$
\frac{\partial \varphi_{k}}{\partial t}=\sum_{k^{\prime}, k^{\prime} \neq k}^{K} \frac{1}{\left|\Omega_{k}\right|}\left[-K L_{w}\left(D\left(\Omega_{k}\right), D\left(\Omega_{k^{\prime}}\right)\right)+\sum_{f=1}^{F} w_{f}^{2}\left(\log \frac{D_{f}\left(\Omega_{k}\right)}{D_{f}\left(\Omega_{k^{\prime}}\right)}-\frac{D_{f}\left(\Omega_{k^{\prime}}\right)}{D_{f}\left(\Omega_{k}\right)}+1\right) * g_{\sigma_{f}}\left(h_{f}(s)\right)\right]\left|\nabla \varphi_{k}\right|
$$




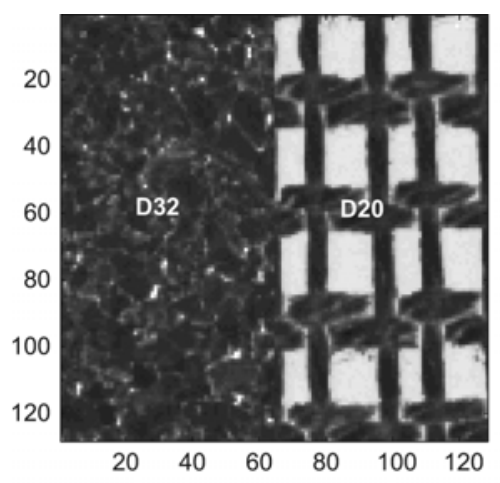

(I1)

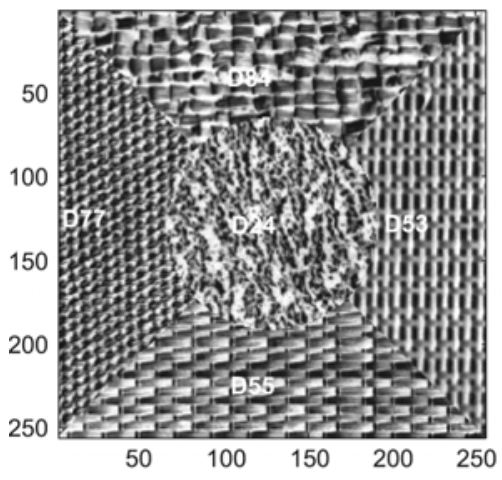

(I3)

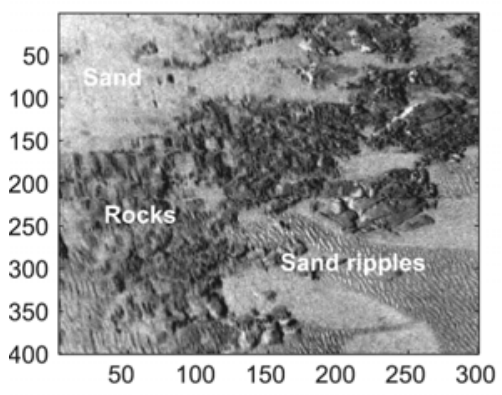

(I5)

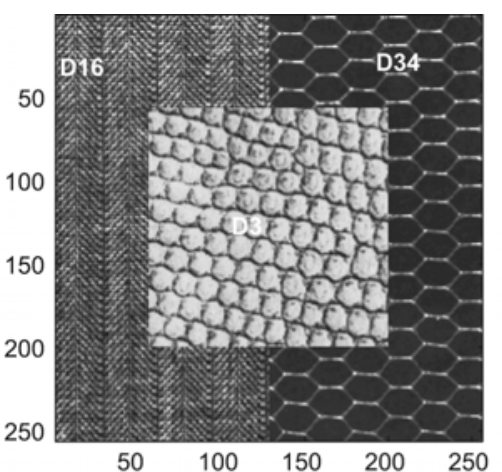

(I2)

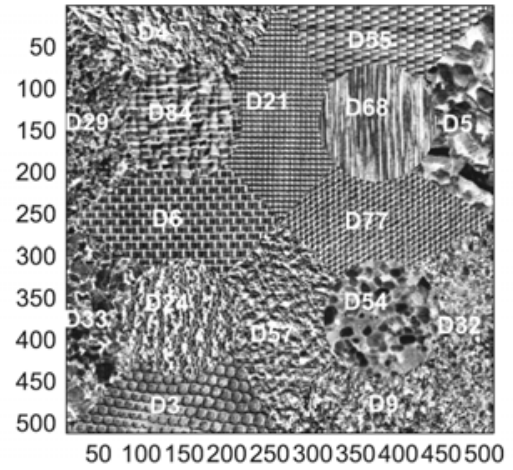

(I4)

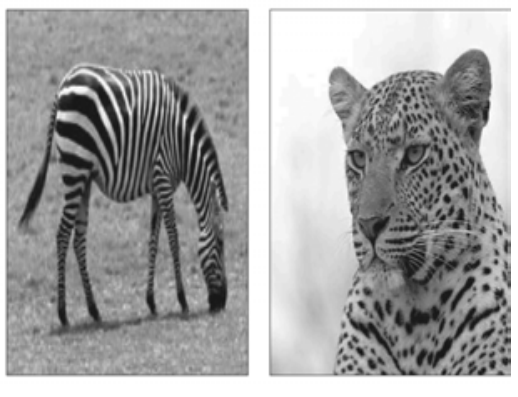

(16) and (17)

Fig. 6. Test images: I1, I2, I3, and I4 are, respectively, 2, 3, 5, and 16 Brodatz mosaics, I4 is a real sidescan image and I6 and I7 are, respectively, natural zebra and leopard images.

Here we consider the fully unsupervised case: neither the class number nor the class prototypes are known. For the estimation of the number of classes we use the method proposed in [33] and [34] based upon a two-term criterion: the intracluster distances and an entropy term. In our case, the intraclass distance is evaluated according to our similarity measure $K L_{w}$.

\section{RESUlTS AND DISCUSSION}

We report segmentation results on four Brodatz texture mosaics [21] with different complexities and on three natural images. The first image, denoted by I1, is a mosaic involving one homogeneous Brodatz-texture (D32) and a coarse Brodatz-texture (D20). The second image I2 is composed of three Brodatz textures (D34, D3, and D16). The third image I3 is a five-texture mosaic with histogram equalized Brodatz-textures (D77, D84, D55, D53 et D24). The fourth image I4 is a more complex mosaic composed of sixteen histogram equalized Brodatz textures (D3, D4, D5, D6, D9, D21, D24, D29, D32, D33, D54, D55,
D57, D68, D77, and D84) and is denoted by I3. The latter two images were used in a comparative study carried out by Randen et al. [28] to evaluate several feature selection methods. Image I5 is a real sidescan sonar image composed of three seafloor types [35]: a coarse texture of rock, an homogeneous class of mud and oriented texture associated to sand ripples (Fig. 6). The last images I6 and I7 are, respectively, a zebra and a leopard image often used in previous works [11], [14], [17], [18].

For the supervised case, for each test image, we first select the most discriminant feature statistics keeping only features that account for more than $90 \%$ of the total weight sum. For image I1, three Gabor energy based distributions computed for parameters $\left(f_{0}, \theta\right) \in$ $\left\{\left(2^{-8} \sqrt{2}, 0^{\circ}\right),\left(2^{-8} \sqrt{2}, 25^{\circ}\right),\left(2^{-5} \sqrt{2}, 135^{\circ}\right)\right\}$ are selected, for image I2, only two Gabor energy based distributions computed for parameters $\left(f_{0}, \theta\right) \in\left\{\left(2^{-6} \sqrt{2}, 0^{\circ}\right),\left(2^{-4} \sqrt{2}, 0^{\circ}\right)\right\}$ are selected with weights 0.45 and 0.42 . For image I3, two co-occurrence distributions computed for $\left(d_{x}, d_{y}\right) \in$ 
TABLE I

SEgmentation ERror RATES (\%) COMPUTED For the PROPOSED AND Classical APPROACHES: MEAN AND STD ARE THE MEAN AND THE STANDARD DEVIATION ERROR RATE

\begin{tabular}{|c||c|c|c|c|c|c|c|}
\hline & $V_{H}: 3$ & $V_{H}: 9$ & $V_{H}: 33$ & $V_{c l}$ & $V_{p S}$ & $V_{p U}$ & Randen \\
\hline I1 & $7 \%$ & $\tau=6$ & 10 & 11 & $\mathbf{2 . 7}$ & - & - \\
\hline I2 & 9 & 5 & 4.6 & 2 & $\mathbf{0 . 9}$ & 1 & - \\
\hline I3 & 70 & 30 & 7 & $\mathbf{3}$ & 4.9 & 5.1 & 8.2 \\
\hline I4 & 80 & 70 & 19 & 63 & $\mathbf{1 3 . 9}$ & 17 & 37.2 \\
\hline I5 & 25 & 13 & 13 & 13.5 & $\mathbf{7 . 5}$ & - & - \\
\hline mean & 38.2 & 24.8 & 10.8 & 18.5 & $\mathbf{5 . 9}$ & 7.7 & 22.7 \\
\hline std & 34.5 & 27.2 & 5.6 & 25.3 & $\mathbf{5}$ & 8.3 & 20.5 \\
\hline
\end{tabular}

$\{(2,1),(6,1)\}$ and a Gabor filter computed for parameters $\left.\left(f_{0}, \theta\right)=\left(2^{-6} \sqrt{2}, 0^{\circ}\right)\right\}$ are selected with weights $0.4,0.3$ and 0.1 . For image I4, the segmentation is carried out using only three co-occurrence matrices computed for parameters: $\left(d_{x}, d_{y}\right) \in\{(0,3),(0,6),(2,2)\}$ with weights 0.25 , 0.25 , and 0.4 . For image $\mathrm{I} 5$, the selected features are the two co-occurrence distributions computed for parameters $\left(d_{x}, d_{y}\right) \in\{(1,4),(2,1)\}$. For all the images, the selection step allows a drastic decrease in the dimension of feature space: an average of three selected features among the initial set of 219 features.

A comparative analysis in terms of classification error rates is reported in Table I. Several approaches are considered.

- The best segmentation results reported in the comparative study on texture feature discrimination power carried out by Randen et al. in [28]. These results are denoted by Randen.

- Classical variational approaches for which we use the energy form proposed by the authors but the texture features issued from our selection/fusion step.

- The Heiler et al. [19] method applied for three different sizes of square neighborhood, namely $T_{W}=3,9$, and 33. This method is denoted by $V_{H}$.

- A variational level set setting, denoted by $V_{c l}$, using a pixel based data-driven energy term (2) with $\Psi()=$. $\sum_{f=1}^{F} w_{f}^{2} \log \left(p_{f}^{k}().\right)$, where $p_{f}^{k}$ is the distribution of texture $k$ response to the filter indexed by $f$. This variational setting is the one exploited in [11] and [14]-[17] but with other texture features.

- The proposed region-based variational setting, denoted by $V_{p S}$ for the supervised case and $V_{p U}$ for the unsupervised case with $w_{f}^{2}=1 / F$.

1) Performance of the Proposed Similarity Measure: Images I3 and I4 were used in [28] for the evaluation of a variety of texture feature selection methods. In [28], Randen et al. perform a comparative study on several filtering approaches including Laws masks, ring/wedge filters, dyadic Gabor filter banks, wavelet transforms, wavelet packets, wavelet frames, quadrature mirror filters, discrete cosine transform, eigenfilters, optimized Gabor filters, linear predictors, and optimized finite impulse response filters and nonfiltering approaches that are based upon co-occurrence and autoregressive features. We obtain better results than the most effective methods reported in [28]. For image I3, the obtained error rate is $4.9 \%$ whereas the best error rate reported in [28] is about $8.2 \%$ and the average classification error rate for all compared methods is about

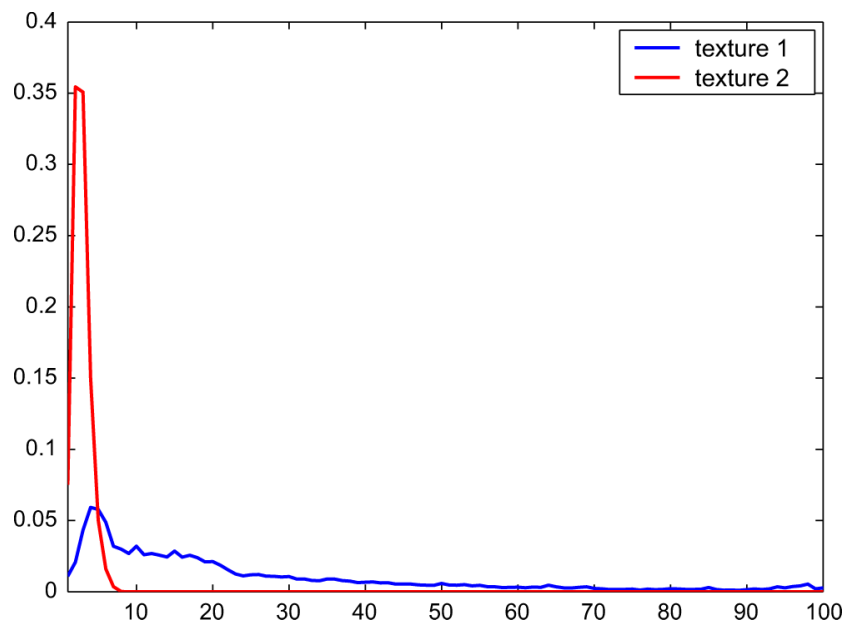

Fig. 7. Gabor energy based distributions for textures of image I1 (texture D32 in red and D68 in blue).

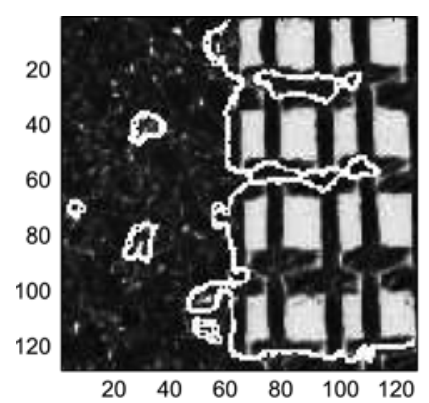

(a)

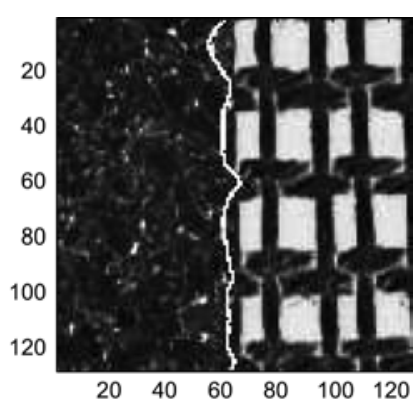

(b)
Fig. 8. Segmentation of image I1. (a) Classical approach $\left(V_{c l}\right)$. (b) Proposed method $\left(V_{p S}\right)$.

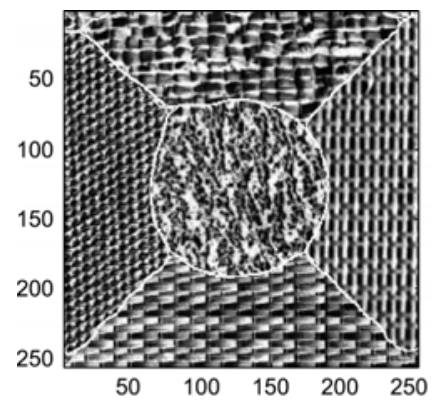

(a)

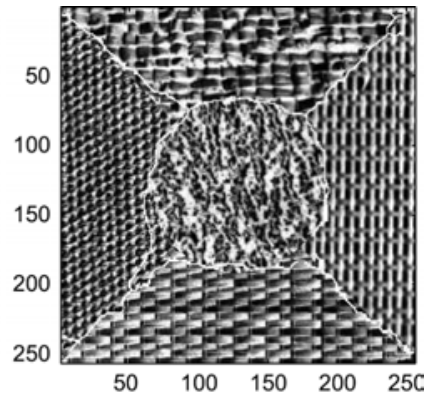

(b)
Fig. 9. Segmentation of image I3. (a) Classical method $\left(V_{c l}\right)$. (b) Proposed approach $\left(V_{p S}\right)$.

$23.6 \%$. For image I4, the error rate for the proposed segmentation is about $14 \%$ and it is about $38 \%$ for the best method evaluated in [28] and the average error rate is $53.8 \%$ for this image. These results stress the relevance of the proposed similarity measure fusing various texture feature types. They also motivate the use of the feature sets issued from this selection step in the implementation of the different variational segmentation settings. More examples are given on our previous work [26].

2) Region-Based $V_{p S}$ Versus Classical Approach $V_{c l}$ : Using the same texture descriptors, segmentation results reported in Table I show that the proposed region-level setting significantly 


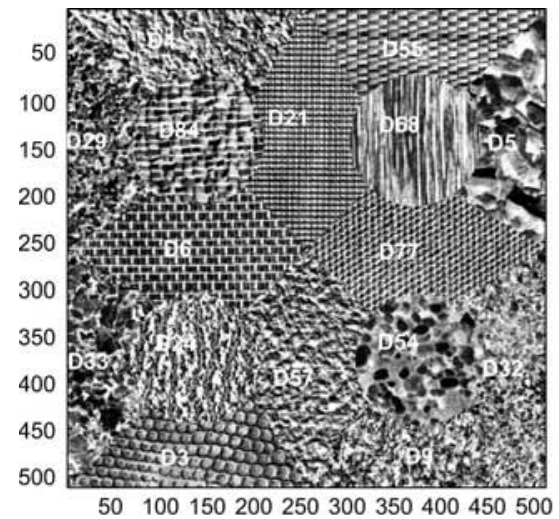

(a)

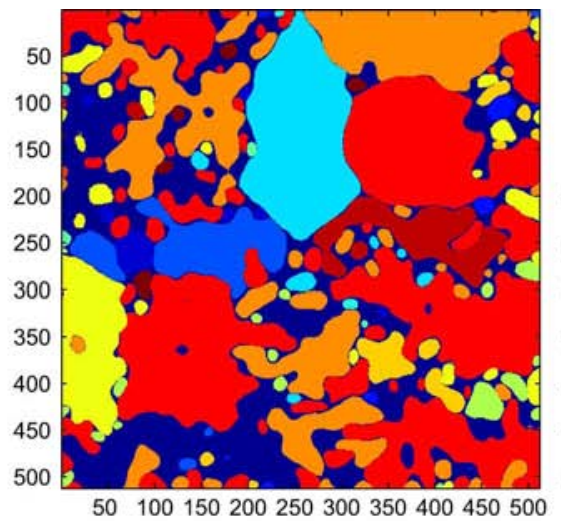

(b)

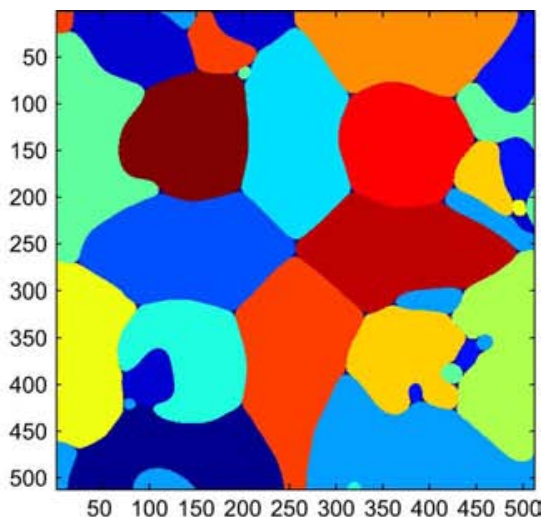

(c)

Fig. 10. Segmentation of image I4. (a) Original image. (b) Classical method $\left(V_{c l}\right)$. (c) Proposed approach ( $\left.V_{p s}\right)$.

outperforms the classical methods $V_{c l}$ : the mean misclassification rate is about $5.9 \pm 5 \%$ for the proposed method ( Var $_{\text {props }}$ ) where it is of $18.5 \pm 25.3 \%$ for $V_{c l}$. Var props significantly outperforms $V_{c l}$ in all cases, except for image I3. Significant improvements are brought in all cases compared to $\operatorname{Var}_{\text {clas }}$, especially $2.7 \%$ versus $11 \%$ and $13.9 \%$ versus $63 \%$ for images I1 and I4, respectively. For image I3, the classical pixel-level variational scheme $V_{c l}$ slightly outperforms the proposed approach (3\% versus $4.9 \%$ ). For this special case, image textures are well seperated in the feature space. In such case, the maximization of the pixel-level likelikood performed by the classical method may achieve a sligtly better detection of the region boundaries than the region-level scheme which seeks to reproduce region feature statistics similar to the reference class statistics. In contrast, when texture classes partially overlap in the considered feature space such as for images I1 and I4, the former strategy is misleading and poor segmentation performances may be achieved. For image I4, the misclassification rate is greater than $60 \%$ for $V_{c l}$ where the region-level criterion $V_{p S}$ reachs a satisfactory misclassification rate of only $13.9 \%$. In the case of image I1, even if the mosaic is simple and the image contains only two textures, the classical method fails in discriminating between textures (Fig. 8) because of the overlap between the selected texture descriptors (Fig. 7).

3) Region-Based Versus Heiler et al. Approach $\left(V_{H}\right)$ : In contrast to classical methods based upon punctual pixel likelihoods $V_{c l}$, Heiler et al. approach exploits pixel neighborhood [19]. Segmentation results show that this method can be more relevant than $V_{c l}$ if we use optimal analysis window size: the mean misclassification rate is about $10.8 \pm 5.6 \%$ for $V_{H}$ with $T_{W}=33$ where it is of $18.5 \pm 25.3 \%$ for $\operatorname{Var}_{\text {clas }}$. However, results issued from $V_{H}$ greatly depend upon the choice of the scale or window parameter: the mean misclassification rate is about $10.8 \pm 5.6 \%$ for $V_{H}$ with $T_{W}=33$ and it is about $38.2 \pm 34.5 \%$ for $T_{W}=3$. Great values of this scale parameter produce better estimates of the texture statistics. But, they can also lead to undesirable situations where multiple texture classes are present in a common window [Fig. 4(c)]. In contrast, smaller analysis windows are less likely to contain multiple classes. But, the limited coverage may, however, produce misleading features [Fig. 4(a)].

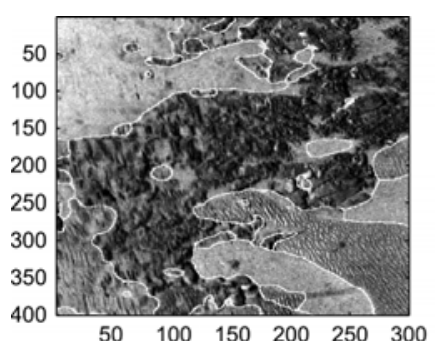

(a)

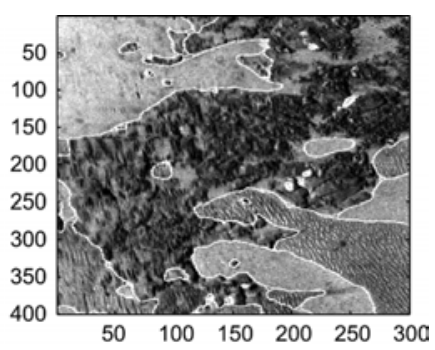

(b)
Fig. 11. Segmentation of image I5. (a) Classical method $\left(V_{c l}\right)$. (b) Proposed approach $\left(V_{p S}\right)$.

The proposed variational region-level segmentation circumvents the problem related to the choice of the neighborhood $W_{s}$ size and accurately detects region boundaries (see Fig. 5) and significant improvements are brought in all cases compared to method $V_{H}$, especially $2.7 \%$ versus $6 \%, 0.9 \%$ versus $4.6 \%$, and $7.5 \%$ versus $13 \%$ for images I1, I2, and I5, respectively.

Overall the performances of the two pixel-level approaches greatly depend upon the potential overlapping of the distributions of the considered feature and on the scale of the texture patterns. In contrast, the proposed region-level approach circumvent these limitations and satisfactory results are reported in all cases.

4) Robustness to Initialization: The proposed variational region-level segmentation is robust to initialization as illustrated in Figs. 12-14, where different initializations for the segmentation of I 2 are considered namely a random initialization, an initialization with a maximum-likelihood segmentation using $T_{W}=3 \times 3$ and an initialization according to the maximum-likelihood segmentation using $T_{W}=33 \times 33$. Similar results (classification error rate of about $1 \%$ ) are obtained in all cases (see http://public.enst-bretagne.fr/rfablet/ Demos/demoIKaroui/Initialization1.avi, http://public.enst-bretagne.fr/rfablet/Demos/demoIKaroui/Initialization2.avi and http://public.enst-bretagne.fr/rfablet/Demos/demoIKaroui/Initialization3.avi).

5) Performance of the Unsupervised Criterion: As in the supervised case, the proposed unsupervised region-level method 


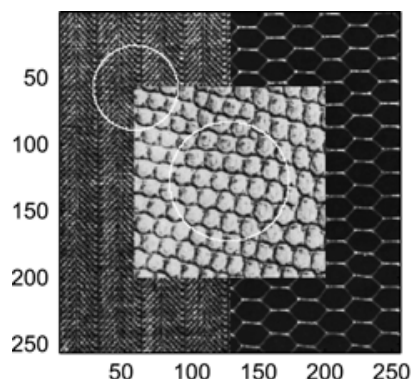

(a)

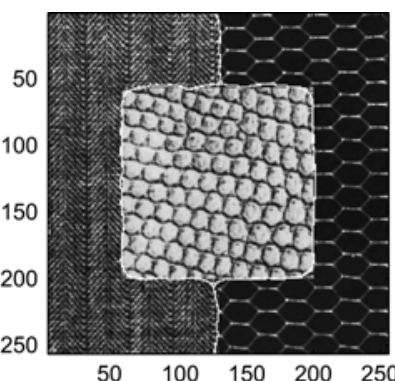

(b)
Fig. 12. Segmentation of image I2 using the proposed approach with a random initialization. (a) Random initialization. (b) Region based segmentation $(\gamma=$ 20,5000 iterations), $\tau=0.9 \%$.

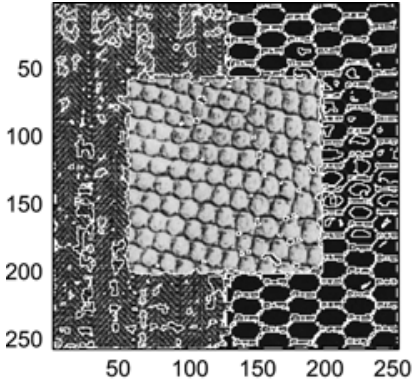

(a) (b)

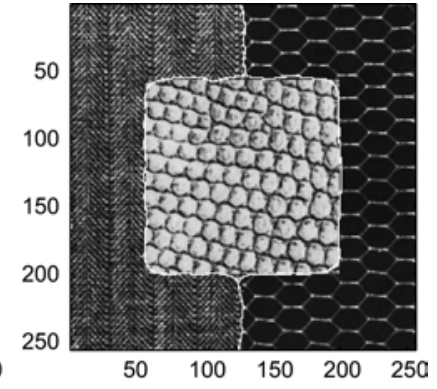

Fig. 13. Segmentation of image I2 using the region-level approach with an initialization issued from a maximum-likelihood criterion. (a) Using a $3 \times 3$ analysis window. (b) Region-level segmentation $\gamma=20,1000$ iterations), $\tau=$ $0.9 \%$

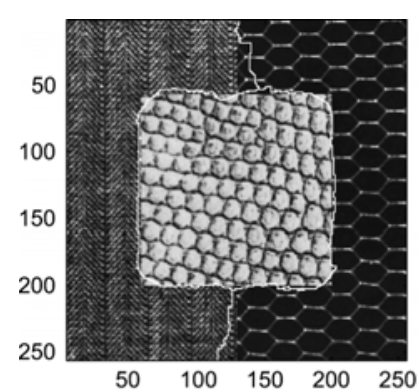

(a)

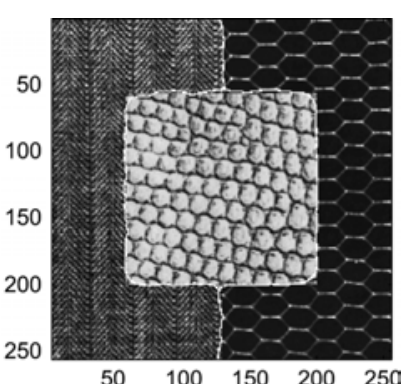

(b)
Fig. 14. Segmentation of image I2 using the region-level approach with an initialization issued from a maximum-likelihood criterion. (a) Using $33 \times 33$ analysis window. (b) Region based segmentation $(\gamma=20,600$ iterations), $\tau=0.9 \%$

is shown to reach good classification performances even compared to classical supervised approaches. For example for the image I2, the error rate with the unsupervised method is about $1 \%$ whereas the average rate is about $5 \%$ for classical supervised approaches (see Table I). Good resulats were obtained for the other images (see Fig. 16).

The unsupervised criterion is however more dependent upon initialization and feature separability than the supervised case. Regarding the latter, the reference statistics are known and the method converges toward regions that conform these statistics regardless of the initialization. In contrast, the unsupervised case does not exploit any reference statistics and the partition that

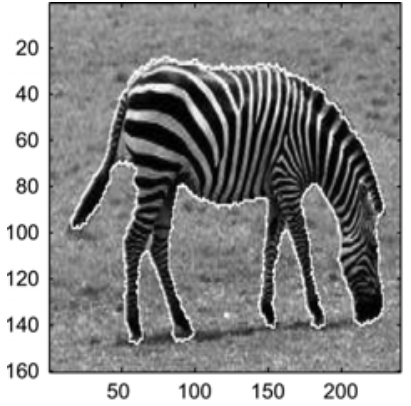

(a)

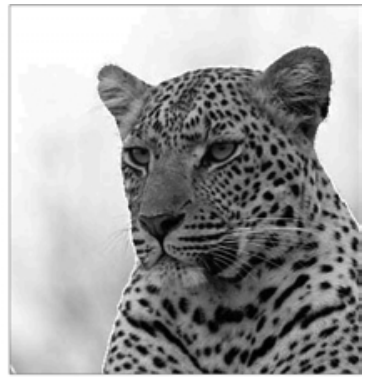

(b)
Fig. 15. Segmentation of natural images I6 and I7 using the proposed regionlevel approach. (a) Segmentation of image I6. (b) Segmentation of image I7.

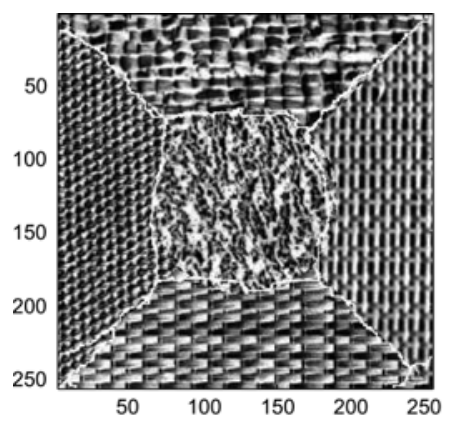

(a)

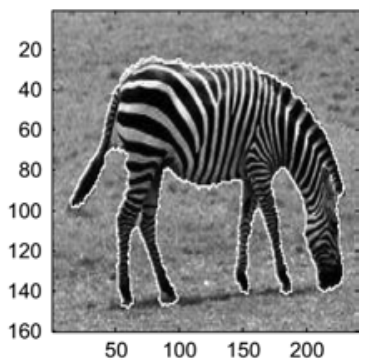

(b)
Fig. 16. Unsupervised segmentation. (a) I3. (b) I6.

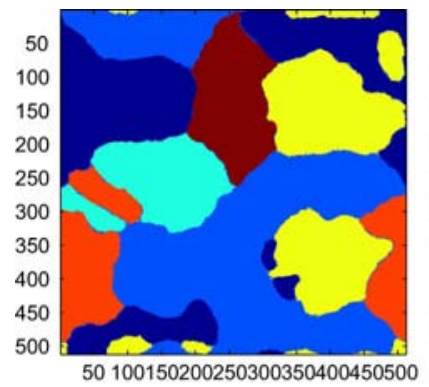

(a)

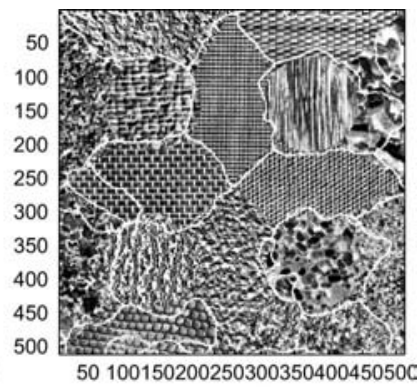

(b)
Fig. 17. Unsupervised segmentation of I4.

maximizes the dissimilarity between region statistics depends upon the separability of region in the chosen feature space. For example, for image I4 (see Fig. 17), only six classes were detected, quite similar textures were grouped:

- D29, D84, D3, and D55;

- D24, D57, D9, D77, and D57;

- D68, D5, and D54;

- D32 and D33.

The segmentation of the mosaic on the six detected classes is homogeneous and the segmentation rate of the proposed unsupervised segmentation is about $60 \%$ (if we consider 16 classes) which is still satisfactory compared to the classical supervised approaches $(80 \%$ for Heiler supervised method using an analyzing window of $9 \times 9$ and pixel-likelihood based approaches $63 \%)$. 


\section{CONCLUSION}

In this paper, we propose and evaluate a region-level variational approach for supervised and unsupervised texture-based segmentation. Texture characterization is issued from nonparametric distributions of their responses to a set of filters. The variational segmentation consists in the minimization of an energy criterion defined over the set of image partitions and comprising two terms: a similarity measure between regions features and texture models and a boundary based functional that imposes smoothness and regularity on region boundaries. The proposed similarity criterion, is generic and permits optimally fusing various types of texture statistics and dealing with different image types. For example, in [27], an application to sidescan sonar images is considered. Experiments were carried out to compare our approach to previous work exploiting variational approaches [2], [3], [11], [14]-[19]. The following conclusions can be drawn.

- The proposed method defines an efficient similarity measure that naturally and optimally fuses a wide variety of texture features.

- The region-based observation-driven term is more robust than pixel-level formulation used in the classical approaches. Existing methods are mainly stated as the optimization of a criterion evaluating punctual pixel likelihoods or similarity measure computed within local neighborhood. These approaches require sufficient dissimilarity between used feature statistics and need the choice of the neighborhood size which may have a considerable impact on segmentation accuracy. The proposed variational setting solves for these two major drawbacks.

In [27], a complementary experimental comparison to Markovian pixel-level segmentation techniques was carried out for sonar images. Similar conclusions were drawn on the superiority brought by the variational region-level setting.

We have shown that the proposed region-level scheme could also be applied to unsupervised image segmentation. The region-level criterion then consists in the dissimilarity maximization between the texture statistics of the different regions. As perspectives for future work, we may mention that the proposed method can integrate automatically other texture features, it can also deal with other computer vision applications such as textured object tracking or detection, etc.

\section{APPENDIX A}

\section{EVOLUTION EQUATION COMPUTATION}

Using the shape derivative tools, we want to differentiate the functional

$$
F\left(\Omega_{k}\right)=\left|\Omega_{k}\right| \sum_{f}^{F} w_{f}^{2} K L\left(Q_{f}^{k}, D_{f}\left(\Omega_{k}\right)\right) .
$$

The Gâteaux derivative of $F\left(\Omega_{k}\right)$ in the direction of a vector field $\vec{V}$ is then given by

$$
\begin{aligned}
& d F\left(\Omega_{k}, \vec{V}\right)=\sum_{f=1}^{F} w_{f}^{2} {\left[d\left[\left|\Omega_{k}\right|\right] K L\left(Q_{f}^{k}, D_{f}\left(\Omega_{k}\right)\right)\right.} \\
&\left.+\left|\Omega_{k}\right| d\left[K L\left(Q_{f}^{k}, D_{f}\left(\Omega_{k}\right)\right)\right]\right] .
\end{aligned}
$$

Replacing $K L$ by its expression, we get

$$
\begin{aligned}
& d\left[K L\left(Q_{f}^{k}, D_{f}\left(\Omega_{k}\right)\right)\right] \\
& \quad=\int_{\alpha} d\left[Q_{f}^{k}(\alpha) \log \left(\frac{Q_{f}^{k}(\alpha)}{D_{f}\left(\Omega_{k}, \alpha\right)}\right) d \alpha\right] \\
& d\left[Q_{f}^{k}(\alpha) \log \left(\frac{Q_{f}^{k}(\alpha)}{D_{f}\left(\Omega_{k}, \alpha\right)}\right) d \alpha\right] \\
& \quad=d\left[Q_{f}^{k} \log \left(Q_{f}^{k}\right)\right]-d\left[Q_{f}^{k} \log \left(D_{f}\left(\Omega_{k}, \alpha\right)\right)\right] .
\end{aligned}
$$

Theorem [25]: The Gâteaux derivative of a functional of the type $K(\Omega)=\int_{\Omega} k(s, \Omega) d s$ is given by

$d K(\Omega, \vec{V})=\int_{\Omega} k_{s h}(s, \Omega, \vec{V}) d s-\int_{\Gamma} k(s, \Omega)(\vec{V} \vec{N}) d a(s)$

where $k_{s h}(s, \Omega, \vec{V})$ is the shape derivative of $k(s, \Omega), d a$ is an area element and $\vec{N}$ the inward unit normal vector of $\Gamma$.

$Q_{f}^{k}$ does not depend upon the domain $\Omega_{k}$, so its Gâteaux derivative is null, and we have

$$
\begin{gathered}
d\left[Q_{f}^{k}(\alpha) \log \left(\frac{Q_{f}^{k}(\alpha)}{D_{f}\left(\Omega_{k}, \alpha\right)}\right) d \alpha\right] \\
=-Q_{f}^{k} d\left[\log \left(D_{f}\left(\Omega_{k}, \alpha\right)\right)\right] \\
d\left[\log \left(D_{f}\left(\Omega_{k}, \alpha\right)\right)\right] \\
=\frac{1}{D_{f}\left(\Omega_{k}, \alpha\right)} d\left[D_{f}\left(\Omega_{k}, \alpha\right)\right] \\
D_{f}\left(\Omega_{k}, \alpha\right)=1 /\left|\Omega_{k}\right| \int_{\Omega_{k}} g_{\sigma_{f}}\left(h_{f}(s)-\alpha\right) d s, \text { so } \\
d\left[D_{f}\left(\Omega_{k}, \alpha\right)\right]=\frac{-1}{\left|\Omega_{k}\right|^{2}} d\left[\left|\Omega_{k}\right|\right] \int_{\Omega_{k}} g_{\sigma_{f}}\left(h_{f}(s)-\alpha\right) d s \\
+\frac{1}{\left|\Omega_{k}\right|} d\left[\int_{\Omega_{k}} g_{\sigma_{f}}\left(h_{f}(s)-\alpha\right) d s\right] \\
=\frac{-1}{\left|\Omega_{k}\right|} D_{f}\left(\Omega_{k}, \alpha\right) d\left[\left|\Omega_{k}\right|\right] \\
+\frac{1}{\left|\Omega_{k}\right|} d\left[\int_{\Omega_{k}} g_{\sigma_{f}}\left(h_{f}(s)-\alpha\right) d s\right] .(18)
\end{gathered}
$$

The Gâteaux derivative of $\left|\Omega_{k}\right|$ is

$$
d\left[\left|\Omega_{k}\right|\right](\vec{V})=-\int_{\Gamma_{k}}(\vec{V} \cdot \vec{N}) d \vec{a}(s) .
$$

As $g_{\sigma_{f}}\left(h_{f}(s)-\alpha\right)$ does not depend upon the domain $\Omega_{k}$, its shape derivative is null. So, we have

$$
\begin{aligned}
d\left[\int _ { \Omega _ { k } } g _ { \sigma _ { f } } \left(h_{f}(s)\right.\right. & -\alpha) d s] \vec{V} \\
& =-\int_{\Gamma_{k}} g_{\sigma_{f}}\left(h_{f}(s)-\alpha\right) \vec{V} \cdot \vec{N} d \vec{a}(s)
\end{aligned}
$$

So

$$
\begin{aligned}
d[ & \left.K L\left(Q_{f}^{k}, D_{f}\left(\Omega_{k}\right)\right)\right] \\
= & \int_{\alpha}\left[\frac{1}{\left|\Omega_{k}\right|} \int_{\Gamma_{k}}\left[\frac{Q_{f}^{k}(\alpha)}{D_{f}(\Omega, \alpha)} g_{\sigma_{f}}\left(h_{f}(s)-\alpha\right)-Q_{f}^{k}(\alpha)\right]\right. \\
& (\vec{V} \cdot \vec{N}) d \vec{a}(s)] d \alpha .
\end{aligned}
$$



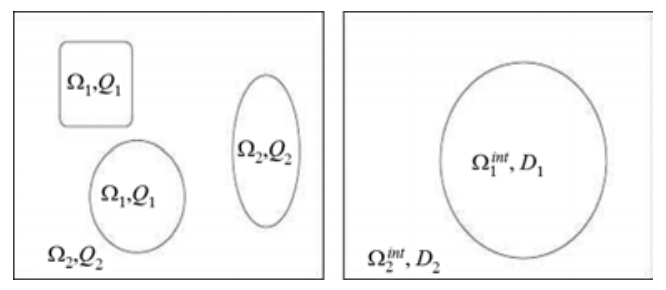

Fig. 18. Left: "true" segmentation. Right: random partition.

We have $\int_{\alpha} Q_{f}^{k}(\alpha) d \alpha=1$, so we get

$$
\begin{aligned}
& d\left[K L\left(Q_{f}^{k}, D_{f}\left(\Omega_{k}\right)\right)\right]=\frac{1}{\left|\Omega_{k}\right|} \\
& \quad \times \int_{\Gamma_{k}}\left[\frac{Q_{f}^{k}}{D_{f}\left(\Omega_{k}\right)} * g_{\sigma_{f}}\left(h_{f}(s)\right)-1\right](\vec{V} \cdot \vec{N}) d \vec{a}(s) .
\end{aligned}
$$

Finally, according to (12), we get

$$
\begin{aligned}
& d F\left(\Omega_{k}, \vec{V}\right)=\int_{\Gamma_{k}}-K L_{w}\left(Q^{k}, D\left(\Omega_{k}\right)\right) \\
& \quad+\sum_{f=1}^{F} w_{f}^{2}\left(\frac{Q_{f}^{k}}{D_{f}\left(\Omega_{k}\right)} * g_{\sigma_{f}}\left(h_{f}(s)\right)-1\right)(\vec{V} \cdot \vec{N}) d \vec{a}(s) .
\end{aligned}
$$

\section{APPENDIX B}

\section{UNSUPERVISED CRITERION}

We show that image partition that maximizes $E^{1}(9)$ corresponds to the "right" segmentation. Without loss of generality we consider the two-class case, the generalization to multiclass case is straightforward. Let $\Omega=\left\{\Omega_{1}, \Omega_{2}\right\}$ be the true partition with statistics $Q_{1}\left(\Omega_{1}\right)$ and $Q_{2}\left(\Omega_{2}\right)$.

For an arbitrarily image partition $\Omega^{\text {int }}=\left\{\Omega_{1}^{\text {int }}, \Omega_{2}^{\text {int }}\right\}$ with region statistics $D\left(\Omega_{1}^{i n t}\right)$ denoted $D_{1}$ and $D\left(\Omega_{2}^{i n t}\right)$ denoted $D_{2}$, (see Fig. 18), we have

$$
D_{1}=\lambda Q_{1}+(1-\lambda) Q_{2}
$$

where $\lambda=\left|\Omega_{1}^{\text {int }} \cap \Omega_{1}\right| /\left|\Omega_{1}^{\text {int }}\right|$ For $D_{2}$ we also have

$$
P_{2}=\lambda^{\prime} Q_{2}+\left(1-\lambda^{\prime}\right) Q_{1}
$$

where $\lambda^{\prime}=\left|\Omega_{2}^{\text {int }} \cap \Omega_{2}\right| /\left|\Omega_{2}^{\text {int }}\right|$. For two class-case, our criterion is the following:

$$
K L\left(D_{1}, D_{2}\right)+K L\left(D_{2}, D_{1}\right) .
$$

The Kullback-Leibler divergence $K L(Q, D)$ is convex with respect to $(Q, D)$ (can easily demonstrated using Jensen inequality), so

$$
\begin{aligned}
& K L\left(D_{1}, D_{2}\right) \\
& \quad=K L\left(\lambda Q_{1}+(1-\lambda) Q_{2}, \lambda D_{2}+(1-\lambda) D_{2}\right) \\
& \quad \leq \lambda K L\left(Q_{1}, D_{2}\right)+(1-\lambda) K L\left(Q_{2}, D_{2}\right)
\end{aligned}
$$

or $K L\left(Q_{1}, D_{2}\right)$ can be expressed as follows:

$$
\begin{aligned}
& \left.K L\left(Q_{1}, D_{2}\right)\right) \\
& \quad=K L\left(\lambda^{\prime} Q_{1}+\left(1-\lambda^{\prime}\right) Q_{1}, \lambda^{\prime} Q_{2}+\left(1-\lambda^{\prime}\right) Q_{1}\right) \\
& \quad \leq \lambda^{\prime} K L\left(Q_{1}, Q_{2}\right)+\left(1-\lambda^{\prime}\right) K L\left(Q_{1}, Q_{1}\right) \\
& \quad \leq \lambda^{\prime} K L\left(Q_{1}, Q_{2}\right) .
\end{aligned}
$$

In the same way

$$
\left.K L\left(Q_{2}, D_{2}\right)\right) \leq\left(1-\lambda^{\prime}\right) K L\left(Q_{2}, Q_{1}\right) .
$$

So

$$
\begin{aligned}
& K L\left(D_{1}, D_{2}\right) \\
& \quad \leq \lambda \lambda^{\prime} K L\left(Q_{1}, Q_{2}\right)+(1-\lambda)\left(1-\lambda^{\prime}\right) K L\left(Q_{2}, Q_{1}\right) .
\end{aligned}
$$

Similarly, we show that

$$
\begin{aligned}
K L\left(D_{2},\right. & \left.D_{1}\right) \\
& \leq \lambda \lambda^{\prime} K L\left(Q_{2}, Q_{1}\right)+(1-\lambda)\left(1-\lambda^{\prime}\right) K L\left(Q_{1}, Q_{2}\right) .
\end{aligned}
$$

Finally, our functional can be majored as follows:

$$
E^{1} \leq\left(\lambda \lambda^{\prime}+(1-\lambda)\left(1-\lambda^{\prime}\right)\right) \times\left(K L\left(Q_{1}, Q_{2}\right)+K L\left(Q_{2}, Q_{1}\right)\right) .
$$

The equality is reached for $\lambda=1$ et $\lambda^{\prime}=1$, i.e., $D_{1}=Q_{1}$ and $D_{2}=Q_{2}$ or for $\lambda=0$ and $\lambda^{\prime}=0$, i.e., $D_{1}=Q_{2}$ and $D_{2}=Q_{1}$.

\section{REFERENCES}

[1] M. N. Do and M. Vetterli, "Texture similarity measurement using Kullback-Leibler distance on wavelet subbands," in Proc. Int. Conf. Image Process., 2000, vol. 3, pp. 730-733.

[2] M. Lianantonakis and Y. R. Petillot, "Sidescan sonar segmentation using active contours and level set method," Oceans-Eur., vol. 1, pp. 719-724, 2005.

[3] T. Kadir and M. Brady, "Unsupervised non-parametric region segmentation using level sets," in Proc. IEEE Int. Conf. Comput. Vis., Oct. 2003, vol. 2, pp. 1267-1274.

[4] L. Xiuwen and W. DeLiang, "Texture classification using spectral histograms," IEEE Trans. Image Process., vol. 12, no. 6, pp. 661-670, Jun. 2003.

[5] R. O. Duda and P. E. Hart, Pattern Classification and Scene Analysis. Hoboken, NJ: Wiley, 1973.

[6] S. Shah and J. K. Aggarwal, "A Bayesian segmentation framework for textured visual images," Comput. Vis. Pattern Recognit., pp. 1014-1020, Jun. 1997.

[7] G. Gimel'farb, "Texture modeling by multiple pairwise pixel interactions," IEEE Trans. Pattern Anal. Mach. Intell., vol. 18, no. 11, pp. 1110-1114, Nov. 1996.

[8] R. Adams and L. Bischof, "Seeded region growing," IEEE Trans. Pattern Anal. Mach. Intell., vol. 16, no. 6, pp. 641-647, Jun. 1994.

[9] P. Nammalwar, O. Ghita, and P. F. Whelan, "Integration of feature distributions for color texture segmentation," in Proc. Int. Conf. Pattern Recognit., Aug. 2005, vol. 1, pp. 716-719.

[10] S. C. Zhu and A. Yuille, "Region competition: Unifying snakes, region growing, and Bayes/MDL for multiband image segmentation," IEEE Trans. Pattern Anal. Mach. Intell., vol. 18, no. 9, pp. 884-900, Sep. 1996.

[11] J. F. Aujol, G. Aubert, and L. Blanc-Féraud, "Wavelet-based level set evolution for classification of textured images," IEEE Trans. Image Process., vol. 12, no. 12, pp. 1634-1641, Dec. 2003.

[12] A. Luminita, A. Vese, and T. F. Chan, "A multiphase level set framework for image segmentation using the Mumford and Shah model," Int. J. Comput. Vis., vol. 50, no. 3, pp. 271-293, 2002. 
[13] C. Samson, L. Blanc-Féraud, G. Aubert, and J. Zerubia, "A level set method for image classification," Int. J. Comput. Vis., vol. 40, no. 3, pp. 187-197, 2000.

[14] N. Paragios and R. Deriche, "Geodesic active regions and level set methods for supervised texture segmentation," Int. J. Comput. Vis., vol. 46, no. 3, pp. 223-247, 2002.

[15] T. Brox and J. Weickert, "Level set based image segmentation with multiple regions," in Pattern Recognition, C. Rasmussen, H. Bulthoff, M. Giese, and B. Scholkopf, Eds. New York: Springer-Verlag, 2004, pp. 415-423.

[16] B. Sandberg, T. Chan, and L. Vese, "A level-set and Gabor-based active contour algorithm for segmenting textured images," Math. Dept., UCLA, Los Angeles, CA, 2002, Tech. Rep. 39.

[17] M. Rousson, T. Brox, and R. Deriche, "Active unsupervised texture segmentation on a diffusion based feature space," in Proc. IEEE Comput. Soc. Conf. Comput. Vis. Pattern Recognit., Jun. 2003, vol. 2 , pp. $699-704$.

[18] S. P. Awate, T. Tasdizen, and R. T. Whitaker, "Unsupervised texture segmentation with nonparametric neighborhood statistics," in Proc. Eur. Conf. Comput. Vis., 2006, pp. 494-507.

[19] M. Heiler and C. Schnorr, "Natural image statistics for natural image segmentation," in Proc. IEEE Int. Conf. Comput. Vis., Oct. 2003, vol. 2, pp. 1259-1266.

[20] J. Kim, J. W. Fisher, and A. Yezzi, "A nonparametric statistical method for image segmentation using information theory and curve evolution," IEEE Trans. Image Process., vol. 14, no. 10, pp. 1486-1502, Oct. 2005.

[21] P. Brodatz, Textures: A Photographic Album for Artists and Designers. New York: Dover, 1996.

[22] O. G. Cula and K. Dana, "3D texture recognition using bidirectional feature histograms," Int. J. Comput. Vis., vol. 49, no. 1, pp. 33-60, Aug. 2004.

[23] Q. Xu, J. Yang, and S. Ding, "Texture segmentation using LBP embedded region competition," Electron. Lett. Comput. Vis. Image Anal., vol. 5, no. 1, pp. 41-47, 2004.

[24] R. Fablet and P. Bouthemy, "Motion recognition using non parametric image motion models estimated from temporal and multiscale cooccurrence statistics," IEEE Trans. Pattern Anal. Mach. Intell., vol. 25, no. 12 , pp. 1619-1624, Dec. 2003.

[25] S. Jehan-Besson, M. Barlaud, and G. Aubert, "Image segmentation using active contours: Calculus of variations or shape gradients?," SIAM J. Appl. Math., vol. 63, no. 6, pp. 2128-2154, 2003.

[26] I. K. Imen, R. Fablet, J. M. Boucher, W. Pieczynski, and J. M. Augustin, "Fusion of textural statistics using a similarity measure: Application to texture recognition and segmentation," Pattern Anal. Appl., vol. 11, no. 3-4, pp. 425-434, Sep. 2008.

[27] I. Karoui, R. Fablet, J. M. Boucher, and J. M. Augustin, "Seabed segmentation using optimized statistics of sonar textures," IEEE Trans. Geosci. Remote Sens., vol. 47, no. 6, pp. 1621-1631, Jun. 2008.

[28] T. Randen and J. H. Husoy, "Filtering for texture classification: A comparative study," IEEE Trans. Pattern Anal. Mach. Intell., vol. 21, no. 4, pp. 291-310, Apr. 1999.

[29] S. Kullback, "On information and sufficiency," in The Annals of Mathematical Statistics. Hoboken, NJ: Wiley, 1951, vol. 22.

[30] J. A. Sethian, Level Set Methods. Cambridge, U.K.: Cambridge Univ. Press, 1996.

[31] A. Herbulot, S. Jehan-Besson, M. Barlaud, and G. Aubert, "Shape gradient for multimodal image segmentation using mutual information," in Proc. Int. Conf. Image Process., 2004, vol. 4, pp. 2729-2732.

[32] T. F. Chan and L. A. Vese, "Active contours without edges," IEEE Trans. Image Process., vol. 10, no. 2, pp. 266-277, Feb. 2001.

[33] G. Palubinskas, X. Descombes, and F. Kruggel, "An unsupervised clustering method using the entropy minimization," in Proc. Int. Conf. Pattern Recognit., 1998, vol. 2, pp. 1816-1818.

[34] A. Lorette, X. Descombes, and J. Zerubia, "Fully unsupervised fuzzy clustering with entropy criterion," in Proc. Int. Conf. Pattern Recognit., 2000, vol. 3, pp. 986-989.

[35] A. Ehrhold, D. Hamon, and B. Guillaumont, "The rebent monitoring network, a spatial integrated acoustic approach to survey nearshore macrobenthic habitats: Application to the bay of concarneau," ICES J. Marine Sci., vol. 63, pp. 1604-1615, 2006.

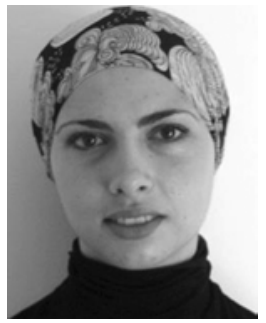

Imen Karoui received the Engineering degree in telecommunications from the Ecole Polytechnique de Tunis, Tunisia, in 2002, the DEA degree in signal, télécommunications, images, and radar from the Université de Rennes 1, Paris, France, in 2003, and the Ph.D. degree in signal processing and telecommunications from the Department Signal and Communications, Telecom Bretagne, Brest Cedex, France, in 2007.

She is currently a Postdoctoral Fellow at Ifremer, Plouzane, France. Her research interests include texture analysis and segmentation and shape recognition and tracking with application to acoustic imaging, fisheries, and oceanography.

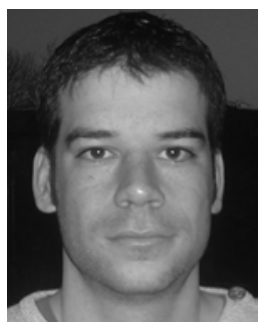

Ronan Fablet received the M.Sc. degree in signal processing and telecommunications from École Nationale Supérieure de 1Aéronautique et de lEspace, Toulouse Cedex 4, France, in 1997, and the Ph.D. degree in signal processing and telecommunications from the University of Rennes 1, Rennes, France, in 2001.

In 2002, he was an INRIA Postdoctoral Fellow at Brown University, Providence. RI. From 2003 to 2007, he had a full-time research position at Ifremer Brest in the field of biological oceanography. Since 2008, he has been an Assistant Professor in the Signal and Communications Department, Telecom Bretagne, Brest Cedex, France. His main interests include sonar and radar imaging especially applied to biological and physical oceanography, and signal and image processing applied to biological archives.

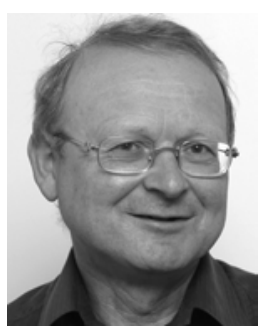

Jean-Marc Boucher (M'83-SM'95) was born in 1952. He received the Engineering degree in telecommunications from the Ecole Nationale Supérieure des Telecommunications, Paris, France, in 1975 and the Habilitationà Diriger des Recherches degree from the University of Rennes 1, Rennes, France, in 1995.

$\mathrm{He}$ is currently a Professor in the Department of Signal and Communications, Telecom Bretagne, Brest Cedex, France, where he is also Education Deputy Director. He is also Deputy Manager of a National Scientific Research Center laboratory (FRE 3167 lab-STICC). His current researches are related to statistical signal analysis including estimation theory, Markov models, blind deconvolution, wavelets, and multiscale image analysis. These methods are applied to radar and sonar images, seismic signals, and electrocardiographic signals. He published about one hundred technical articles in these areas in international journals and conferences.

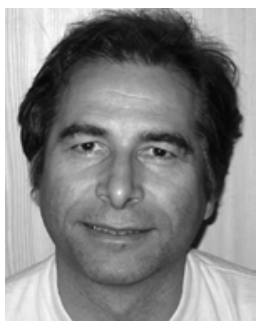

Jean-Marie Augustin received the M.S. degree in signal processing from the University of Rennes, France, in 1982.

He joined the Institut Français de Recherche et dExploitation de la Mer, Brest, France in 1984, and is presently a Senior Engineer in the Department of Acoustics and Seismics. His main interests include software development for sonar seafloor mapping and backscatter reflectivity analysis. 